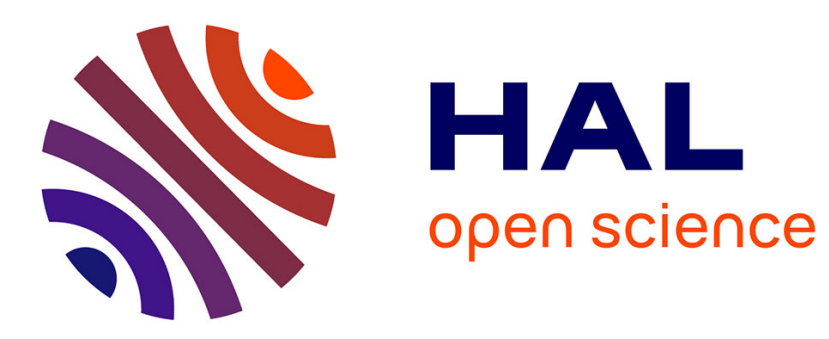

\title{
Nanoscale electrical analyses of axial-junction GaAsP nanowires for solar cell applications
}

Omar Saket, Chalermchai Himwas, Valerio Piazza, Fabien Bayle, Andrea Cattoni, Fabrice Oehler, Gilles Patriarche, Laurent Travers, Stéphane Collin, François H Julien, et al.

\section{To cite this version:}

Omar Saket, Chalermchai Himwas, Valerio Piazza, Fabien Bayle, Andrea Cattoni, et al.. Nanoscale electrical analyses of axial-junction GaAsP nanowires for solar cell applications. Nanotechnology, 2020, 31 (14), pp.145708. 10.1088/1361-6528/ab62c9 . hal-03031146

\section{HAL Id: hal-03031146 https://hal.science/hal-03031146}

Submitted on 30 Nov 2020

HAL is a multi-disciplinary open access archive for the deposit and dissemination of scientific research documents, whether they are published or not. The documents may come from teaching and research institutions in France or abroad, or from public or private research centers.
L'archive ouverte pluridisciplinaire HAL, est destinée au dépôt et à la diffusion de documents scientifiques de niveau recherche, publiés ou non, émanant des établissements d'enseignement et de recherche français ou étrangers, des laboratoires publics ou privés. 


\title{
Nanoscale electrical analyses of axial-junction GaAsP nanowires for solar cell applications
}

\author{
Omar SAKET ${ }^{1}$, Chalermchai HIMWAS $^{1,2}$, Valerio PIAZZA ${ }^{1}$, Fabien BAYLE ${ }^{1}$, Andrea CATTONI ${ }^{1}$, Fabrice \\ OEHLER ${ }^{1}$, Gilles PATRIARCHE ${ }^{1}$, Laurent TRAVERS ${ }^{1}$, Stephane COLLIN ${ }^{1}$, François H. JULIEN ${ }^{1}$, \\ Jean-Christophe HARMAND ${ }^{1}$, Maria TCHERNYCHEVA ${ }^{1}$ \\ 1: Centre de Nanosciences et de Nanotechnologies, UMR 9001 CNRS, Univ. Paris Sud, Univ. Paris-Saclay, 10 Boulevard \\ Thomas Gobert, 91120 Palaiseau Cedex, France.
}

2: Semiconductor Device Research Laboratory, Department of Electrical Engineering, Faculty of Engineering, Chulalongkorn University, 254 Phayathai Road, Bangkok 10330, Thailand

\begin{abstract}
Axial $p-n$ and $p-i-n$ junctions in $\mathrm{GaAs}_{0.7} \mathrm{P}_{0.3}$ nanowires are demonstrated and analysed using electron beam induced current microscopy. Organized self-catalysed nanowire arrays are grown by molecular beam epitaxy on nanopatterned Si substrates. The nanowires are doped using Be and Si impurities to obtain $\mathrm{p}$ - and n-type conductivity, respectively. A method to determine the doping type by analysing the induced current in the vicinity of a Schottky contact is proposed. It is demonstrated that for the applied growth conditions using $\mathrm{Ga}$ as a catalyst, Si doping induces an n-type conductivity contrary to the GaAs self-catalysed nanowire case, where Si was reported to yield a $p$-type doping. Active axial nanowire $p$-n junctions having a homogeneous composition along the axis are synthesized and the carrier concentration and minority carrier diffusion lengths are measured. To the best of our knowledge, this is the first report of axial $p$-n junctions in self-catalyzed GaAsP nanowires.
\end{abstract}

\section{Introduction}

Today, the record in photovoltaic (PV) conversion efficiency is held by three and four junction tandem devices based on III-V semiconductors [1,2]. However, the implementation of these devices is very complex and expensive. To simplify the architecture and to benefit from the maturity of Si photovoltaics, a strong effort is dedicated to the development of III-V on Si two junction tandems $[3,4,5]$. The optimal bandgap for III-V on Si tandem devices is close to $1.7 \mathrm{eV}[3,6]$. Among different III$V$ semiconductors, GaAsP has recently emerged as a promising material for the top subcell: it demonstrates a record efficiency of $15.3 \%$ for GaAsP films grown on a Si(100) substrate, projecting monolithic tandem devices to an overall efficiency higher than 25\% [5]. Nevertheless, the growth of high-quality III-V thin films on Si remains very challenging due to the large defect density induced by the lattice mismatch between the materials. To overcome this issue, an architecture replacing the thin film geometry of the top cell by nanowires (NWs) was introduced $[7,8,9]$.

III-V semiconductor NWs have a number of advantages for PV devices. Due to their free lateral surface, NWs can relax strain without forming dislocations, so that defect-free structures can be grown on lattice-mismatched substrates and in particular on Si [10]. In addition, NW arrays are characterized by a small optical reflectance and enhanced light diffusion, leading to an increased absorption with respect to thin films $[11,12]$.

Currently, NW solar cells demonstrate a record efficiency of $15.3 \%$ for bottom-up GaAs NWs [13] and $17.8 \%$ for top-down InP NWs [14] and their efficiency is continuously growing. A first prototype of a III-V NW on Si tandem cell showing a voltage addition has been reported [15]. However, most of the reported NW PV devices (including the above-mentioned GaAs/Si tandem cell [15]) are based on binary GaAs or InP compounds $[13,16,14]$, while the performance of ternary alloy devices remains far behind. 
Recently, several investigations have targeted the synthesis of ternary (e.g. GaAsP or InGaP) NW arrays with an optimal bandgap for III-V on Si tandems $[17,18,19,20,21]$ and GaAsP NW radial junction solar cells have been reported $[22,23,24,25]$. Still the control over the composition and doping of the ternary alloy NWs remains a major challenge. In some cases, doping incorporation may affect the crystal structure and composition of the NWs [21]. To push forward the efficiency of ternary alloy NW solar cells, it is important to assess both the composition and the electrical properties of NWs down to the nanometric scale level.

The doping profile is an important parameter for optimizing the solar cell efficiency. In the case of 2D layers, the carrier concentration can be easily assessed (e.g. by Hall effect, capacitance-voltage or field effect measurements $[26,27,28])$. However, doping calibrations performed on thin films cannot be easily extrapolated to the NWs because the growth conditions and the incorporation mechanisms are different. For example, silicon which is a conventional n-dopant in planar GaAs (001) layers, is reported to produce a p-doping in GaAs NWs grown by the Vapor Liquid Solid (VLS) mechanism [29]. Due to their nanoscale diameter, doping quantification in NWs is not straightforward. The electrical method using Hall effect [30] yields a high accuracy, but requires a very complex fabrication procedure. The most widespread electrical technique is based on field effect mobility measurements [31,32], however it also requires several processing steps to define the source, drain and gate and its final accuracy critically depends on the approximation errors in the computation of device capacitance. As an alternative, optical contact-free techniques have been developed: Terahertz conductivity spectroscopy (for a review see [33]), Raman spectroscopy [34,35,36], photoluminescence [36] and cathodoluminescence [37]. Most of these methods rely on the measurements of the peak energy and lineshape against a known reference. In the case of binary compounds, a high accuracy can be obtained, whereas doping measurements of ternary alloys by optical methods remain challenging, since spectral modifications induced by doping and alloy variations cannot be unambiguously disentangled.

Electron Beam Induced Current (EBIC) microscopy [38, 39] is an effective alternative to be used for doping investigation in NWs $[40,41,20]$. This technique consists in exposing a semiconductor to an electron beam, which results in the generation of electron-hole pairs within a small interaction volume. The carriers are drifted apart in presence of the electric field and are collected in an external circuit creating an induced current $[38,39,42]$. The doping can be extracted from the measured width of the depletion region [40]. Electrical contacts for EBIC can be provided by nanomanipulators without complex lithographic and metallization steps, which allows for a high characterization throughput [20].

In this contribution, we analyze the electrical properties of self-catalyzed organized $\mathrm{GaAs}_{0.7} \mathrm{P}_{0.3} \mathrm{NWs}$ using EBIC microscopy. First, the doping type in NWs homogeneously doped with Be or $\mathrm{Si}$ is assessed. We propose a method to investigate the doping type by analyzing the induced current polarity in the vicinity of a Schottky contact. It is demonstrated that for the applied growth conditions using Ga as a VLS catalyst, Si behaves as an n-type dopant (contrary to the reports on self-catalyzed VLS GaAs NWs [29]). Next, axial $p-n$ and $p-i-n$ junction NWs are investigated. Energy dispersive $X$-ray spectroscopy (EDX) analyses in a transmission electron microscope (TEM) show no influence of the doping on the alloy composition. The EBIC maps of the NWs present an unambiguous signature of an active $p-n$ junction and allow to estimate the electron and hole concentrations. To the best of our knowledge, this is the first report of axial p-n junctions in self-catalyzed GaAsP NWs.

\section{Sample elaboration}

Axial junction GaAsP NWs were synthesized on patterned $\mathrm{n}^{++}$-type Si (111) substrates by Molecular Beam Epitaxy (MBE). For the substrate patterning, hexagonal arrays of nanoholes with a hexagon side of $500 \mathrm{~nm}$ were defined by electron beam lithography in a 30-nm-thick silica $\left(\mathrm{SiO}_{2}\right)$ layer deposited by plasma-enhanced chemical vapor deposition on the Si substrate. To transfer the nanohole patterns 
into the $\mathrm{SiO}_{2}$ layer, a two-step procedure consisting of a reactive ion etching followed by wet etching was applied. Subsequently, the patterned substrates were outgassed in ultra-high vacuum at $450{ }^{\circ} \mathrm{C}$ for one hour prior to the NW growth [43].

The growth of GaAsP NWs was performed at $610^{\circ} \mathrm{C}$ following the VLS mechanism catalyzed by Ga droplets, i.e. in a self-catalyzed growth mode. The growth was started by a short deposition of $\mathrm{Ga}$ at the growth temperature to form a liquid Ga droplet in the mask openings similar to the procedure described in [43]. Then the Arsenic (As) and Phosphorus (P) shutters were opened simultaneously to initiate the GaAsP NW growth. The Ga flux was set equivalent to a two-dimensional deposition rate on GaAs (001) of $0.2 \mathrm{~nm} \cdot \mathrm{s}^{-1}$, as calibrated from reflection high-energy electron diffraction oscillations. Group V materials ( $A$ s and $P$ ) were supplied by solid-source cells in the form of $A s_{4}$ and $P_{2}$. Both cells were equipped with individual valves and shutters. The $V$-to-III beam equivalent pressure (BEP) ratio was set at 9 while the $P_{2} /\left(P_{2}+A s_{4}\right)$ BEP ratio was adjusted to 0.2 . The resulting $P$ content in the GaAsP alloy deduced from EDX analyses was close to $30 \%$. The axial NW growth rate was in the $1.45 \mathrm{~nm} / \mathrm{s}$ to $1.8 \mathrm{~nm} / \mathrm{s}$ range.

$\mathrm{Be}$ and $\mathrm{Si}$ sources were used as dopant species. The temperature of the $\mathrm{Si}(\mathrm{Be})$ cell was set to 1150 ${ }^{\circ} \mathrm{C}\left(700^{\circ} \mathrm{C}\right)$. As calibrated on GaAs (001) planar films grown at a deposition rate of $0.2 \mathrm{~nm} \cdot \mathrm{s}^{-1}$, these cell temperatures yield respectively $n$-doped ( $p$-doped) GaAs layers with a doping concentration of $6 \times 10^{18}$ $\mathrm{cm}^{-3}\left(2 \times 10^{18}\right)$ according to Hall measurements. By opening and closing the doping cell shutters during the GaAsP NW growth, homogenously doped NWs and NWs containing axial p-n or p-i-n junctions were synthesized. Homogeneously doped wires had a $\sim 0.55 \mu \mathrm{m}$ height and a base diameter $\sim 50 \mathrm{~nm}$. The $p-n$ junctions were formed by growing first the $n$-doped segment and then switching without growth interruption to the p-doped segment (the total NW height was $\sim 1.3-1.4 \mu \mathrm{m}$ with nominally identical $n$ - and $p$ - doped segments and a base diameter $\sim 70 \mathrm{~nm}$ ). For the $\mathrm{p}$-i-n junction NWs, a $\mathrm{n}$ doped segment, an unintentionally doped segment and a p-doped segment were grown in a sequence without growth interruptions (the total NW height was $\sim 1.6-1.5 \mu \mathrm{m}$ with nominally identical $n-, \mathrm{i}-$ and p- doped segments and a base diameter $\sim 85 \mathrm{~nm}$ ).

Figure 1 a) displays an SEM image of the p-i-n GaAsP NW array. It shows that NWs are highly homogeneous and present a good verticality with a low density of defects in the pattern (about $80 \%$ of vertical NWs).
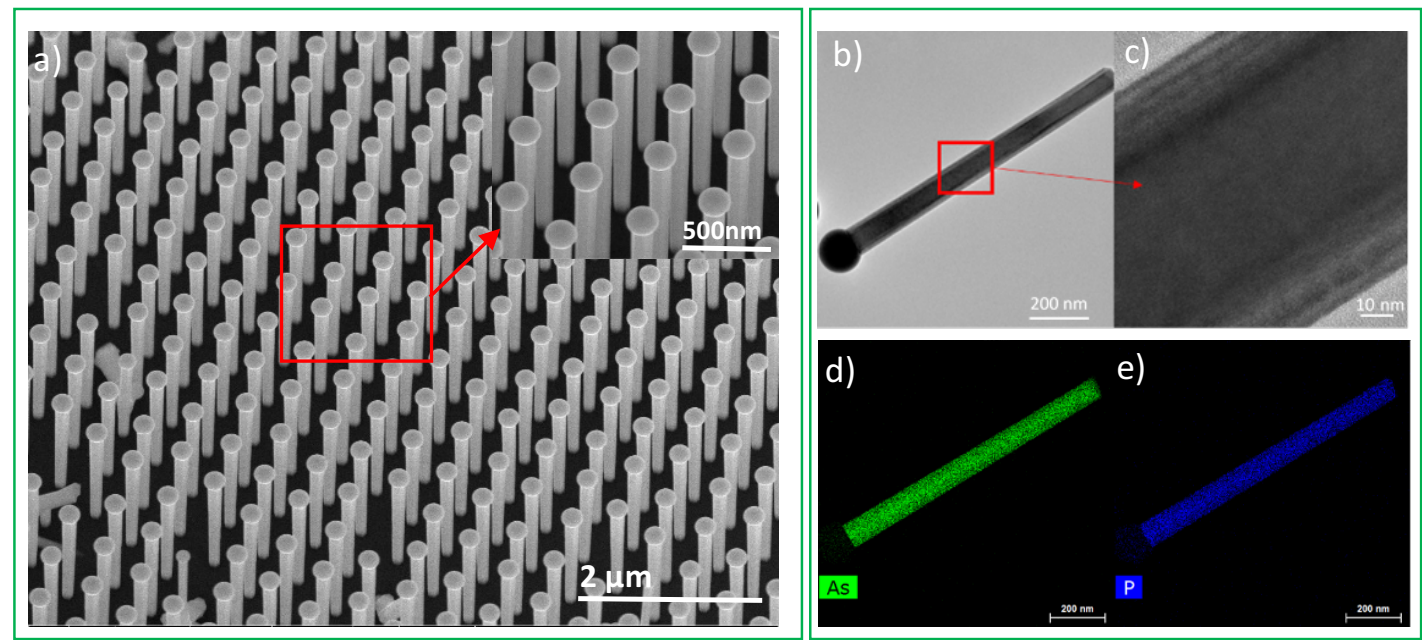

Figure 1 a) $45^{\circ}$ tilted SEM image of GaAsP NW array. Inset shows a high magnification image of the same sample; $b$ ) STEM image of a GaAsP NW containing a p-i-n junction; c) high magnification STEM image of the same NW; d) EDX maps of the arsenic concentration along the NW; e) Same for the phosphorous concentration.

It has been reported that the crystal structure and composition of ternary alloy NWs can be affected by doping incorporation (e.g. p-doping with Zn in MOVPE-grown InGaP NWs [21]). To assess the structure and composition of our NWs, individual NWs were transferred to a TEM grid and were analysed in a FEI Titan Themis microscope operated in scanning TEM (STEM) mode and equipped with 
EDX tool. Figures $1 \mathrm{~b}$ ) and c) show STEM images of a GaAsP NW containing a p-i-n junction. The crystal structure was analysed in high resolution in the n-doped, undoped and $\mathrm{p}$-doped parts of the NW (an example of a high-resolution image is displayed in panel 1c). All segments have a zinc-blende structure with few twin defects. No significant modification of the twin density in the three segments was observed. In addition, EDX compositional maps were acquired. Figures $1 \mathrm{~d}$ ) and e) display the measured $\mathrm{P}$ and As concentration. The composition is homogenous along the $\mathrm{NW}$ axis; it is equal to $\mathrm{GaAs}_{0.7} \mathrm{P}_{0.3}$ (we note that this value is measured on the NW sidewall, so it gives a compositional average over any eventual radial variations, which may appear in ternary NWs $[44,45])$. We conclude that in the MBE grown GaAsP NWs, the dopant $(\mathrm{Be}, \mathrm{Si})$ incorporation does not influence the crystal quality or NW composition.

\section{Doping type investigation}

First, the doping type in homogeneously doped NWs was determined. For (001)-oriented GaAsP thin films, Be (Si) doping results in a p-type (n-type) material [46]. However, in NWs the growth mechanism is different with respect to thin films. The adatoms in a VLS growth are incorporated through the Ga liquid catalyst. In these conditions, the incorporation of doping impurities can strongly differ from the thin film case grown using group $\mathrm{V}$ overpressure. In addition, $\mathrm{Si}$ is an amphoteric dopant, i.e. its electrical behavior depends on its incorporation site. In particular, Si was reported to behave as a p-type dopant in Ga-catalyzed GaAs NWs [29]. For this reason, alternative doping impurities such as Tellurium were explored for $n$-doping of GaAs NWs [36]. However, no investigations of Si doping type in self-catalyzed ternary GaAsP NWs have been reported so far. Therefore, we first analyze the doping type in homogeneously-doped GaAsP NWs to select appropriate doping impurities for the $p$-n junction formation.

To investigate the doping type, we analyzed homogeneously-doped GaAsP NWs by EBIC microscopy. Indeed, EBIC maps allow to detect the internal fields [47]. Therefore, to determine the doping type we analyze the direction of the induced current in the proximity of a Schottky contact which is voluntarily created using a nanomanipulator. Figures 2 a) and d) show schematic representations of the electron beam exciting a Schottky contact on a n-type and a p-type semiconductor, respectively. The difference between the two cases is the direction of the internal electric field. For the n-type semiconductor, the bands bend upwards (figure $2 \mathrm{~b}$ )) which should lead to a positive induced current, while for the $\mathrm{p}$-type semiconductor the bands bend downwards (figure 2 e)) and a negative current should be produced.

To perform this assessment, the Schottky contact between the NW body and the catalyst Ga particle contacted with a tungsten tip was analyzed. Single NW EBIC characterization was performed using a Hitachi SU8000 SEM with a probe station PS4 from Kleindiek equipped with 4 nanomanipulators with tungsten tips (diameter $\sim 200 \mathrm{~nm}$ ). The induced current was amplified with a Stanford SR570 preamplifier, the point by point acquisition of the EBIC maps was controlled with a Gatan DigiScan system. As a result, the SEM image of the scanned surface and the corresponding induced current cartography were acquired simultaneously. For all measurements, the acceleration voltage was fixed at $7 \mathrm{kV}$ and the impinging current was $\sim 55 \mathrm{pA}$. During the EBIC acquisition, an external $\mathrm{DC}$ bias ranging from $-5 \mathrm{~V}$ to $+5 \mathrm{~V}$ was applied to the NWs. This allows to change the band bending in the structure (e.g. to flatten or enhance the electric field at the semiconductor/metal interface) and thus to improve the signal to noise ratio in cases where the internal electric field is weak. 

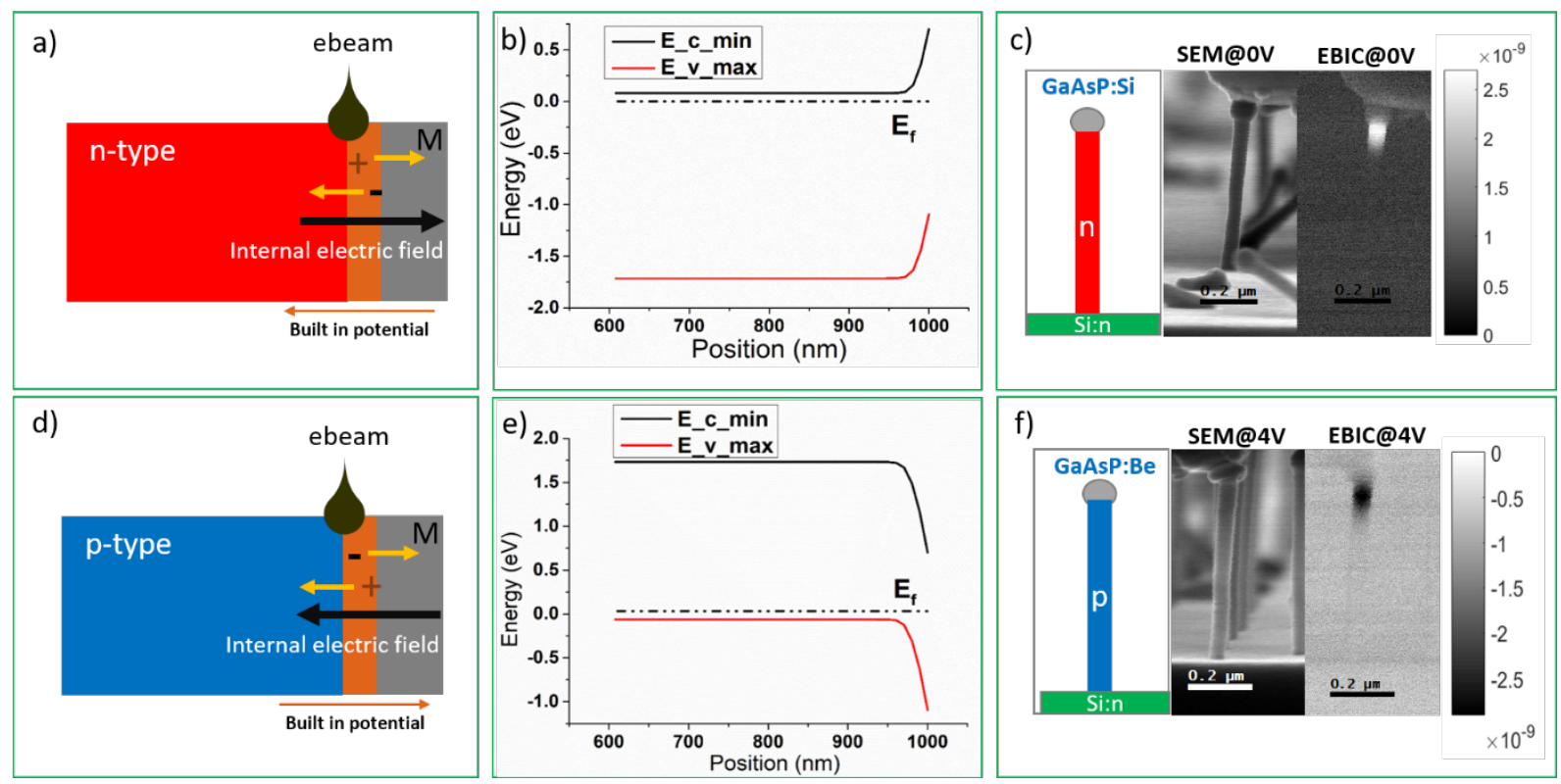

Figure.2 a) Schematic of the e-beam excitation of a Schottky contact on a n-type semiconductor; b) Nextnano [48] simulation of the corresponding band bending calculated for a barrier height of $0.7 \mathrm{~V}$; c) Schematic, SEM image and the correspond EBIC map of a GaAsP NW homogenously doped with Si (color scale bar in Amps); d) Schematic of the e-beam excitation of a Schottky contact on a p-type semiconductor; e) Nextnano simulation of the corresponding band bending calculated for a barrier height of $0.7 \mathrm{~V}$; f) Schematic, SEM image and the corresponding EBIC map of a GaAsP NW homogenously doped with Be (color scale bar in Amps).

In EBIC maps (figures $2 \mathrm{c}$ ) and f)) the direction of the current is coded by the colour contrast: the dark contrast corresponds to a negative current while the bright contrast indicates a positive current. In figure $2 \mathrm{c}$ ) we report the EBIC results for a Si doped GaAsP NW. The figure shows an SEM image of an individual NW contacted via the catalyst droplet on the top with a tungsten tip. The corresponding EBIC map collected under zero bias is also shown. A bright contrast is observed at the interface between the catalyst droplet and the NW, which is a signature of a Schottky contact on an n-type semiconductor. This proves that contrary to the GaAs NWs case [29], Si doping in Ga-catalysed ternary GaAsP NWs can result in n-type conductivity. The same method was applied to the Be doped NWs, as illustrated in figure $2 \mathrm{f}$ ). For this case, the EBIC signal at zero external bias was below the noise level. To enhance the contrast, an external bias was applied. Under negative bias no induced current was observed. However, under a positive bias, a dark EBIC contrast was evidenced localized at the interface between the catalyst and the NW. An example of the EBIC map under $+4 \mathrm{~V}$ external bias is shown in figure $2 \mathrm{f}$ ). The positive external bias is expected to enhance the downward band bending at the semiconductor/catalyst interface. The positive sign of the bias and the dark EBIC contrast observed in this case is the signature of a Schottky contact on a p-type semiconductor (in agreement with the usual behaviour of the Be doping [36]). It should be noted that a similar sample containing self-assembled Be-doped NWs exhibited a dark EBIC contrast both under positive and under zero bias, which further confirms that Be produces a p-type doping in GaAsP Ga-catalyzed NWs.

\section{EBIC characterisation of GaAsP nanowires containing a p-n junction}

After demonstrating the possibility to obtain $p$ - and $n$ - doping in NWs, axial $p-n$ junctions were grown and analyzed. EBIC maps were collected for individual NWs contacted on the catalyst droplet. Figure 3 a) displays a schematic, an SEM image and an EBIC map of an individual p-n junction NW. From the EBIC map the electrical activity of the junction can be visualized without any external bias. To determine the position of the $p$-n junction, secondary electrons (SE) contrast in the SEM image is used 
[40]. This contrast is barely distinguishable without external bias, but can be enhanced under reverse bias [49] (it can be clearly seen in the SEM image of figure 3 a) acquired at an external bias of $-4 \mathrm{~V}$ ).

The IV characteristic of the same NW under identical contact configuration was acquired using a Keithley 2636 sourcemeter (with a blanked electron beam). The curve displayed in figure $3 \mathrm{~b}$ ) shows a rectifying behaviour expected for a $p-n$ junction. It exhibits a leakage current increasing with reverse bias, which may arise from the surface conductivity [50].
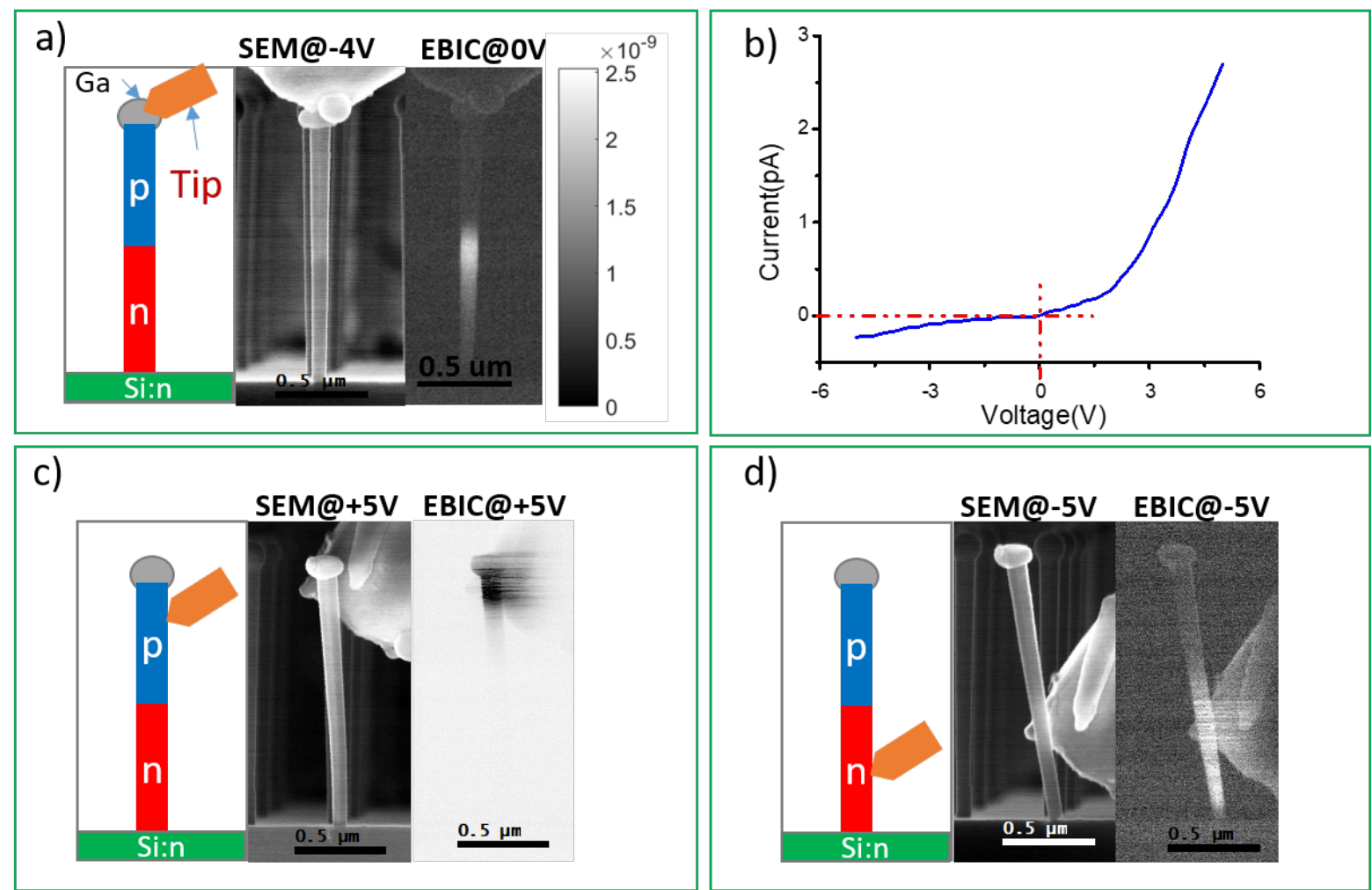

d)

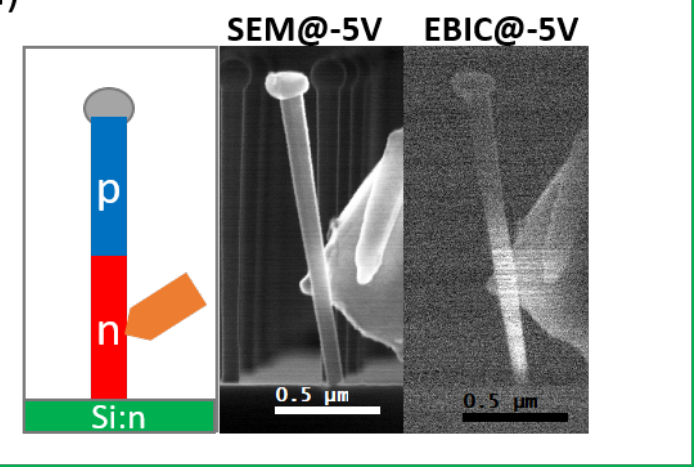

Figure.3.a) Schematic, SEM image and EBIC map of an individual NW containing a p-n junction contacted on the top (color scale bar in Amps); b) Single NW IV characteristic in the dark; c) Same images as in panel a) for the same NW laterally contacted in the top p-doped part; d) Same images as in panel a) for the same NW laterally contacted in the bottom ndoped part.

Nano-manipulators enable EBIC measurements with a lateral contact at different positions along the NW. Here a lateral contacting was used to confirm the doping type of the top and bottom NW segments following the Schottky contact evaluation method described in the previous section. First, the tip was placed laterally on the top segment above the junction and a forward bias was applied (figure $3 \mathrm{c}$ )). In these conditions, the bright EBIC contrast of the junction disappeared (the built-in field was flattened by the external bias) and a dark EBIC contrast appeared in the vicinity of the contact with the tungsten tip. This dark contrast is an indication of a Schottky contact on a p-type semiconductor in agreement with the nominal structure. Then, the tip was placed laterally close to the NW base, a negative bias was applied and a bright EBIC contrast was observed, which confirms that the base part is $n$-doped. These experiments validate the nominal structure of the axial $p$ - $n$ junction.

In order to extract the carrier concentration and the minority carrier diffusion lengths, the axial EBIC profile should be analysed. This profile is theoretically modelled as a convolution of the generation function with the probability of charge collection [51]: 


$$
I_{E B I C}(x)=h(x) * Q(x)
$$

where $I_{E B I C}(x)$ is the induced current, $h(x)$ is the spatial distribution of the generation, and $Q(x)$ is the probability of carrier collection. This latter can be approximated as unity inside the depletion layer. Outside this region, the charge collection probabilities can be approximated as [51] :

$$
Q(x)=e^{-\frac{x}{L_{d}}},
$$

where $L_{d}$ is the diffusion length of the corresponding minority carriers.

Combining expressions (1) and (2), the induced current is divided into three parts: the diffusion current in the p-doped segment, the drift current in the depletion layer and the diffusion current in the $n$ doped segment. The well-known diffusion current expression is given by:

$$
I_{\text {diff }}(x)=I_{\text {max }} e^{-\frac{x}{L_{d}}}
$$

Therefore, the minorities carrier diffusion lengths can be obtained by fitting the decay of the induced current in the $p$ and $n$ parts far from the depletion region with an exponential function. The analysis of the EBIC profile inside and close to depletion region is more complex. Indeed, the EBIC profile strongly depends on the spatial distribution of the generation function and its overlap with the depletion region $[51,52,53]$. For excitation close to the depletion region edge it is difficult to separate the drift and the diffusion components of the current [51,52]. To localize the space charge region edges with a high accuracy, the deconvolution of the EBIC profile with the spatial distribution of the generation should be computed. In this case, the generation function need to be calculated (for example, using beammatter interaction model based on a Monte-Carlo simulation of electron trajectories, which is not straightforward for the considered complex 3D geometry of free-standing NWs). To avoid the computation complexity, we have adopted another method to determine the depletion region extension proposed by Kurniawan [51], which consists in computing the second derivative of the natural logarithm of the induced current close to the depletion region edge and localising the minimum point. We note that for the experimental EBIC profile the measurement noise compromises the accuracy of both methods since this noise is amplified by making the deconvolution or by taking the second derivative. This inaccuracy results in important error bars for the extracted values of the depletion region edges, as seen below.

To determine separately the carrier concentration in the $\mathrm{p}$ - and $\mathrm{n}$-doped segments, the respective extension of the depleted region in two semi-junctions should be determined, which requires to localize the junction position. This can be done by analysing the SE contrast [40]. To localise the junction interface with a high precision, the noise of the experimental SE profile is filtered out and the position of the junction interface is determined by the local minimum of its first derivative. Figure 4 displays the SE contrast under a reverse bias of $-4 \mathrm{~V}$, the filtered SE contrast, the derivative of the filtered SE contrast, and the EBIC profile under zero bias along the NW axis. The junction is localized at the minimum of the derivative of the SE contrast, which lies within the region with the highest EBIC current (cf. figure $4 \mathrm{a}$ )). For the displayed NW, the position of the junction is located at $0.63 \pm 0.01 \mu \mathrm{m}$ from the NW base.

By combining the SEM and the EBIC profile analyses, the space charge region extension in the $p$ and in the $n$ parts is measured. For the representative NW analysed in figure 4, the total depletion region width is estimated to be around $95 \pm 38 \mathrm{~nm}$. Taken into account the error on the position of the depletion layer edge and on the junction interface position, the extension of the space charge region is estimated to be $39 \pm 14 \mathrm{~nm}$ in the $p$ part and $52 \pm 16 \mathrm{~nm}$ in the $\mathrm{n}$ part These values were used to estimate the doping concentrations following the approach detailed in $[40,20]$. The electron and hole concentrations were estimated to be around $6.7 \pm 3.3 \times 10^{17} \mathrm{~cm}^{-3}$ and $9.3 \pm 4.9 \times 10^{17} \mathrm{~cm}^{-3}$, respectively. 
The error bars correspond to the doping levels calculated for the maximum and for the minimum extension of the depletion region in the $\mathrm{n}$ - and $\mathrm{p}$ - parts. The high value of the error bars is mostly related to the uncertainty in the determination of the depletion region edges by the method of Kurniawan [51] and to a smaller extent to the uncertainty of the junction position. Several NWs from the same pattern were analysed to evaluate the wire to wire variations of the $W_{p}$ and $W_{n}$, however statistical deviation was below the above-mentioned uncertainty of the method.

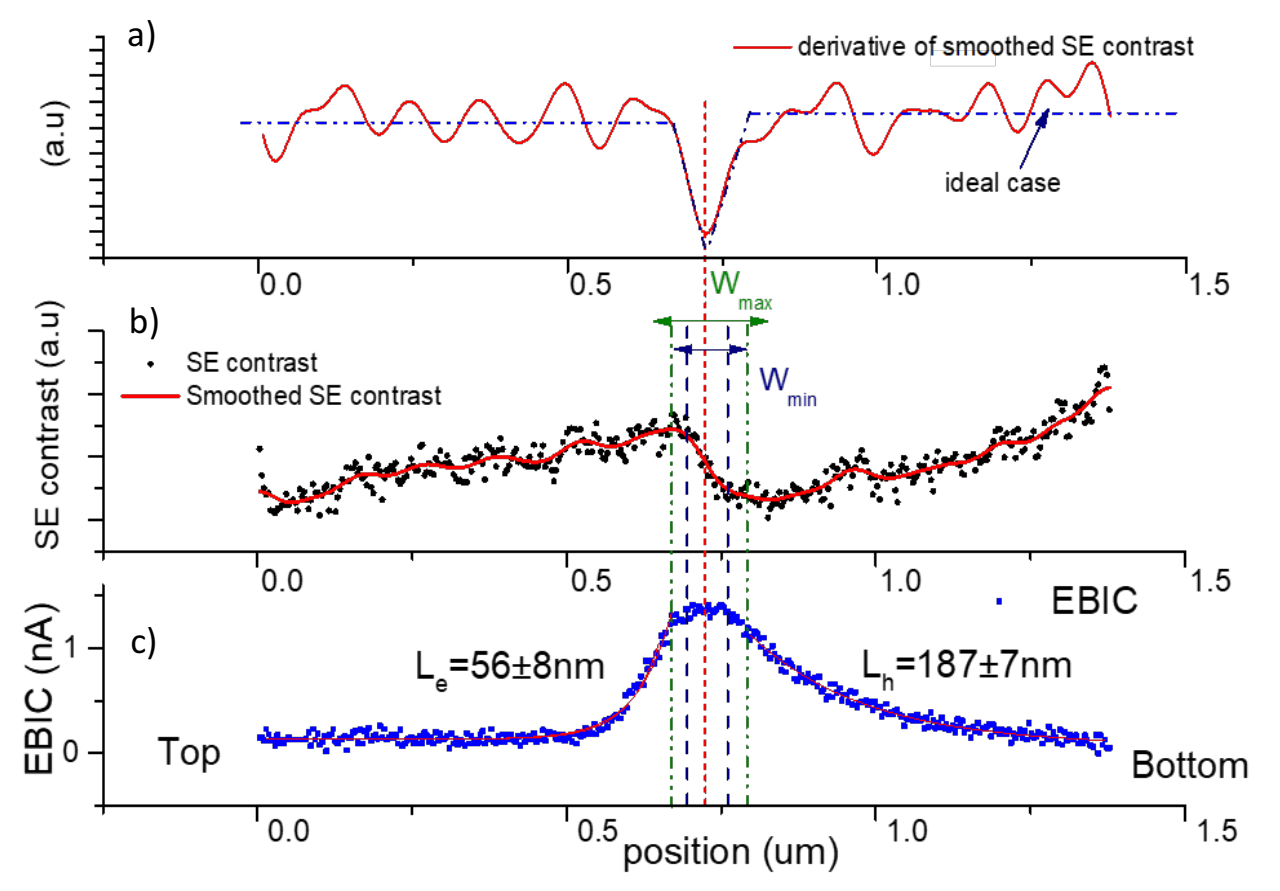

\footnotetext{
Figure.4.a) Derivative of the SE contrast (the blue dashed-dotted line shows the ideal case); b) SE contrast profile under $-4 \mathrm{~V}$ external bias $\left(W_{\max }\right.$ and $W_{\min }$ are respectively the maximum and the minimum of the estimated depletion region width); c) EBIC profile under zero bias along the NW axis, the red line is the exponential fit of the current decay in the NW. To reduce the noise, all line profiles are averaged over a $20 \mathrm{~nm}$ wide region in the direction normal to the wire axis.
}

The extracted carrier concentrations show that for the chosen growth conditions and temperatures of the doping cells, a $\mathrm{p}$-n junction with well-balanced $\mathrm{p}$ - and $\mathrm{n}$ - doping levels is achieved. To compare the extracted carrier concertation in the NW with the doping concentration measured on the reference 2D GaAs films using Hall effect, a corrective factor arising from the growth rate difference should be applied. For the considered example, the average NW growth rate is $1.75 \mathrm{~nm} / \mathrm{s}$ (to be compared with $0.2 \mathrm{~nm} / \mathrm{s}$ growth rate used for the growth of the reference thin films). By applying a corrective factor related to the difference in the growth rates, the doping concentration extrapolated from the thin film case would be $6.8 \times 10^{17} \mathrm{~cm}^{-3}$ for the electrons and $2.3 \times 10^{17} \mathrm{~cm}^{-3}$ for the holes, respectively. The extracted electron concentration in the $\mathrm{n}$-doped segment of the NW is consistent with the extrapolation from the thin film value. The hole concentration in the p-doped segment is higher, however it remains within the right order of magnitude. We note that the reliability of the estimation based on thin film doping values is limited because of the strong difference in growth conditions between the NWs and the thin films. 
To extract the minority carrier diffusion length, the current decay outside the depletion layer is fitted with an exponential function. The electron diffusion length in the $p$-doped segment is estimated to be around $56 \pm 8 \mathrm{~nm}$ and the hole diffusion length in the $\mathrm{n}$-doped segment is around $187 \pm 7 \mathrm{~nm}$. These small diffusion lengths can be explained by a high surface recombination in these non-passivated NWs [54]. Similarly, short diffusion lengths were reported in the literature for GaAs NW p-n junctions (namely, $130 \pm 2 \mathrm{~nm}$ for electrons and $150 \pm 3 \mathrm{~nm}$ for holes [41]).

\section{EBIC characterisation of GaAsP nanowires containing an axial $p$-i-n junction}

Due to the high surface recombination, the carrier collection region of the $p-n$ junction NWs is very narrow and cannot be applied to solar cell devices. To extend the active region, a $p$-i-n junction was designed with doped $p$ - and $n$ - extremities having the same doping concentration as for the $p-n$ junction case and a nominally undoped region in between. The structure was grown and characterized by EBIC and the results are illustrated in figure 5 .

Figure 5 a) shows a NW schematic, an SEM image and a corresponding EBIC map under zero applied bias. A bright EBIC contrast is observed in the lower part of the NW, which confirms the electrical activity of the p-i-n junction. However, contrary to our expectations, the EBIC signal is not covering the entire length of the intrinsic region. Contrariwise, the signal is localized close to the interface between the $\mathrm{n}$-doped and the nominally intrinsic segments. This means that the undoped GaAsP part exhibits a p-type behaviour. To investigate the effective doping type of the intrinsic region, EBIC measurements with a lateral contact at different positions along the NW were performed as shown in fig. 5 b), c) and d). The contact positions are located in the 3 different regions, namely $p$-doped, intrinsic and $n$-doped segments. In the first case, a dark EBIC contrast was observed under a forward bias (shown in fig. 5 b)), while under zero or reverse bias a bright contrast was evidenced in the lower part of the wire at the same position as in the fig. 5 a) case (not shown for the sake of space). These observations are consistent with flattening of the junction potential under forward bias, in which case only the Schottky band bending produces an induced current. Under zero or reverse bias the junction signal is collected for the lateral contact similarly to the top contact case. Then the tip was positioned laterally on the nominally intrinsic region and the experiment was repeated (Fig. $5 \mathrm{c}$ )). The observations were identical to those for the contact on the p-doped part. Namely, a dark EBIC contrast was observed under a positive bias, however with a stronger noise level. This indicates that the nominally undoped GaAsP segment presents a residual $p$-doping. This unintentional $p$-doping may arise from the Be incorporation by radial growth or by Be diffusion [55] during the growth of the top Be-doped segment. Finally, the bottom $\mathrm{n}$-doped segment was laterally contacted (Fig. 5 d)). In this case, a bright EBIC contrast arising from a Schottky contact with a tungsten tip is observed (only under reverse bias) localized close to the contact position in agreement with the nominal $n$-doping of the base segment.

The EBIC profile of the structure (Fig. 5 e) shows that despite the undesired p-type behaviour of the intrinsic region, the space charge region of the $p$-i-n junction is extended in comparison with the p-n junction pointing out to a lower effective $p$-doping in the intrinsic segment compared to the intentionally Be-doped segment. A two-fold increase is observed with the space charge region width equal to $240 \pm 40 \mathrm{~nm}$. 
Fig. $5 \mathrm{f}$ ) shows the IV characteristics of the same NW contacted on the top measured in dark and under electron beam exposure in scanning mode. The IV curve shows a rectifying behaviour typical of a diode with a reverse leakage current independent of the bias lower than $0.1 \mathrm{pA}$. The IV under electron beam irradiation shows a measurable short circuit current and an open circuit voltage estimated around $0.8 \mathrm{~V}$. Under forward bias, the dark and illuminated IV characteristics have the same slope,
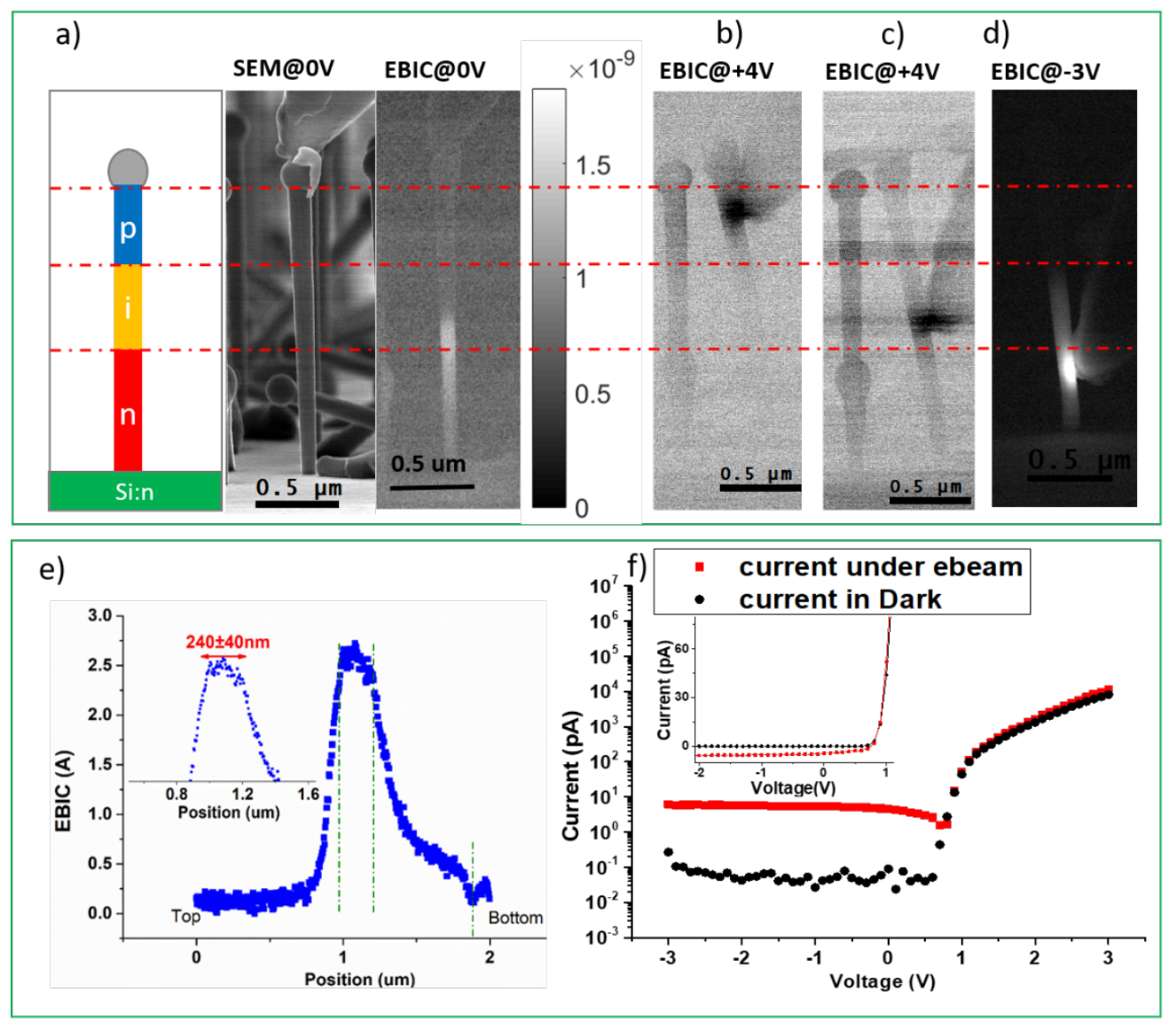

Figure.5.a) Schematic, SEM image and corresponding EBIC map of an individual NW containing a p-i-n junction contacted on the top (color scale bar in Amps); EBIC map of the same NW contacted laterally b) on the top p-segment; $c$ ) on the intrinsic region; d) on the bottom $n$-segment; e) The EBIC profile along the NW axis, Inset is the zoom on the flat EBIC signal; f) I-V characteristics in logarithmic scale of the NW in the dark and under electron beam irradiation (insert shows the I-V curve in linear scale).

which in particular shows the absence of photoconductive effects in NW doped extremities.

\section{Conclusion}

To summarize, we have demonstrated active axial p-n and p-i-n junctions in $\mathrm{GaAs}_{0.7} \mathrm{P}_{0.3} \mathrm{NWs}$ and analyzed their electrical properties using EBIC microscopy. Self-catalyzed NW arrays have been grown by a vapour-liquid-solid method on Si patterned substrates by Molecular Beam Epitaxy. First, the doping type has been assessed by analyzing a Schottky contact on NWs homogeneously doped with Be or Si. We have concluded that for self-catalysed VLS grown GaAsP NWs, Be is a p-type dopant while $\mathrm{Si}$ is an $\mathrm{n}$-type dopant. Then EBIC has been used to probe the electrical activity of the axial GaAsP p-n and $p$-i-n junctions. Based on the EBIC profile across the junction, the carrier concentrations have been estimated to be $6.7 \pm 3.3 \times 10^{17} \mathrm{~cm}^{-3}$ for the electrons and $9.3 \pm 4.9 \times 10^{17} \mathrm{~cm}^{-3}$ for the holes. In order to extend the carrier collection region, a $\mathrm{p}$-i-n junction has been elaborated. The EBIC analyses of the $\mathrm{p}$ $\mathrm{i}-\mathrm{n}$ NWs have demonstrated that the nominally intrinsic segment presented a residual $p$-type doping, leading to an internal field localization close to the $n / i$ interface. 
[1] Tauzin A, Lagoutte E, Salvetat T, Guelfucci J, Bogumilowicz Y, Imbert B, Fournel F, Reboh S, Luce F P, Lecouvey C, Chaira T, Carron V, Moriceau H, Duvernay J, Signamarcheix T, Drazek C, Charles-Alfred C, Ghyselen B, Guiot E, Tibbits T, Beutel P and Dimroth F 2015 InP-based composite substrates for four junction concentrator solar cells AIP Conference Proceedings 1679040009

[2] Dimroth F, Tibbits T N D, Niemeyer M, Predan F, Beutel P, Karcher C, Oliva E, Siefer G, Lackner D, Fuß-Kailuweit P, Bett A W, Krause R, Drazek C, Guiot E, Wasselin J, Tauzin A and Signamarcheix T 2016 Four-Junction Wafer-Bonded Concentrator Solar Cells IEEE Journal of Photovoltaics 6 343-9

[3] Essig S, Ward S, Steiner M A, Friedman D J, Geisz J F, Stradins P and Young D L 2015 Progress Towards a 30\% Efficient GaInP/Si Tandem Solar Cell Energy Procedia 77 464-9

[4] Soga T, Baskar K, Kato T, Jimbo T and Umeno M 1997 MOCVD growth of high efficiency current-matched AlGaAsSi tandem solar cell Journal of Crystal Growth 174 579-84

[5] Vaisman M, Fan S, Nay Yaung K, Perl E, Martín-Martín D, Yu Z J, Leilaeioun M, Holman Z C and Lee M L 2017 15.3\%-Efficient GaAsP Solar Cells on GaP/Si Templates ACS Energy Lett. 2 1911-8

[6] Almansouri I, Ho-Baillie A, Bremner S P and Green M A 2015 Supercharging Silicon Solar Cell Performance by Means of Multijunction Concept IEEE Journal of Photovoltaics 5 968-76

[7] LaPierre R R 2011 Theoretical conversion efficiency of a two-junction III-V nanowire on Si solar cell Journal of Applied Physics 110014310

[8] Bu S, Li X, Wen L, Zeng X, Zhao Y, Wang W and Wang Y 2013 Optical and electrical simulations of two-junction III-V nanowires on Si solar cell Appl. Phys. Lett. 102 031106

[9] Borgström M T, Magnusson M H, Dimroth F, Siefer G, Höhn O, Riel H, Schmid H, Wirths S, Björk M, Åberg I, Peijnenburg W, Vijver M, Tchernycheva M, Piazza V and Samuelson L 2018 Towards Nanowire Tandem Junction Solar Cells on Silicon IEEE Journal of Photovoltaics 8 733-40

[10] Glas F 2006 Critical dimensions for the plastic relaxation of strained axial heterostructures in free-standing nanowires Phys. Rev. B 74121302

[11] Zhu J, Yu Z, Fan S and Cui Y 2010 Nanostructured photon management for high performance solar cells Materials Science and Engineering: R: Reports 70 330-40

[12] Muskens O L, Rivas J G, Algra R E, Bakkers E P A M and Lagendijk A 2008 Design of Light Scattering in Nanowire Materials for Photovoltaic Applications Nano Lett. 8 $2638-42$ 
[13] Åberg I, Vescovi G, Asoli D, Naseem U, Gilboy J P, Sundvall C, Dahlgren A, Svensson K E, Anttu N, Björk M T and Samuelson L 2016 A GaAs Nanowire Array Solar Cell With 15.3\% Efficiency at 1 Sun IEEE Journal of Photovoltaics 6 185-90

[14] van Dam D, van Hoof N J J, Cui Y, van Veldhoven P J, Bakkers E P A M, Gómez Rivas J and Haverkort J E M 2016 High-Efficiency Nanowire Solar Cells with Omnidirectionally Enhanced Absorption Due to Self-Aligned Indium-Tin-Oxide Mie Scatterers ACS Nano 10 11414-9

[15] Yao M, Cong S, Arab S, Huang N, Povinelli M L, Cronin S B, Dapkus P D and Zhou C 2015 Tandem Solar Cells Using GaAs Nanowires on Si: Design, Fabrication, and Observation of Voltage Addition Nano Lett. 15 7217-24

[16] Wallentin J, Anttu N, Asoli D, Huffman M, Åberg I, Magnusson M H, Siefer G, FussKailuweit P, Dimroth F, Witzigmann B, Xu H Q, Samuelson L, Deppert K and Borgström M T 2013 InP Nanowire Array Solar Cells Achieving 13.8\% Efficiency by Exceeding the Ray Optics Limit Science 339 1057-60

[17] Zhang Y, Wu J, Aagesen M, Holm J, Hatch S, Tang M, Huo S and Liu H 2014 SelfCatalyzed Ternary Core-Shell GaAsP Nanowire Arrays Grown on Patterned Si Substrates by Molecular Beam Epitaxy Nano Lett. 14 4542-7

[18] Dastjerdi M H T, Boulanger J P, Kuyanov P, Aagesen M and LaPierre R R 2016 Methods of Ga droplet consumption for improved GaAs nanowire solar cell efficiency Nanotechnology 27475403

[19] Himwas C, Collin S, Rale P, Chauvin N, Patriarche G, Oehler F, Julien F H, Travers L, Harmand J-C and Tchernycheva M 2017 In situ passivation of GaAsP nanowires Nanotechnology 28495707

[20] Tchoulfian P, Donatini F, Levy F, Dussaigne A, Ferret P and Pernot J 2014 Direct Imaging of $\mathrm{p}-\mathrm{n}$ Junction in Core-Shell GaN Wires Nano Lett. 14 3491-8

[21] Otnes G, Heurlin M, Zeng X and Borgström M T 2017 InxGa1-xP Nanowire Growth Dynamics Strongly Affected by Doping Using Diethylzinc Nano Lett. 17 702-7

[22] Holm J V, Jørgensen H I, Krogstrup P, Nygård J, Liu H and Aagesen M 2013 Surfacepassivated GaAsP single-nanowire solar cells exceeding 10\% efficiency grown on silicon Nature Communications 41498

[23] Zhang Y, Aagesen M, Sanchez A M, Beanland R, Wu J and Liu H 2017 GaAsP nanowires and nanowire devices grown on silicon substrates Quantum Sensing and Nano Electronics and Photonics XIV Quantum Sensing and Nano Electronics and Photonics XIV vol 10111 (International Society for Optics and Photonics) p 101110X

[24] Tchernycheva M, Rigutti L, Jacopin G, Bugallo A de L, Lavenus P, Julien F H, Timofeeva M, Bouravleuv A D, Cirlin G E, Dhaka V, Lipsanen H and Largeau L 2012 Photovoltaic properties of GaAsP core-shell nanowires on $\mathrm{Si}(001)$ substrate Nanotechnology 23265402

[25] Holm J V, Aagesen M, Zhang Y, Wu J, Hatch S and Liu H 2014 Bandgap optimized III-V (GaAsP) nanowire on silicon tandem solar cell, device and data 2014 IEEE 40th 
Photovoltaic Specialist Conference (PVSC) 2014 IEEE 40th Photovoltaic Specialist Conference (PVSC) pp 1041-4

[26] Götz W, Kern R S, Chen C H, Liu H, Steigerwald D A and Fletcher R M 1999 Halleffect characterization of III-V nitride semiconductors for high efficiency light emitting diodes Materials Science and Engineering: B 59 211-7

[27] Ambridge T and Faktor M M 1975 An automatic carrier concentration profile plotter using an electrochemical technique $J$ Appl Electrochem 5 319-28

[28] Jarrett C P, Friend R H, Brown A R and de Leeuw D M 1995 Field effect measurements in doped conjugated polymer films: Assessment of charge carrier mobilities Journal of Applied Physics 77 6289-94

[29] Dufouleur J, Colombo C, Garma T, Ketterer B, Uccelli E, Nicotra M and Fontcuberta i Morral A 2010 P-Doping Mechanisms in Catalyst-Free Gallium Arsenide Nanowires Nano Lett. 10 1734-40

[30] Hultin O, Otnes G, Borgström M T, Björk M, Samuelson L and Storm K 2016 Comparing Hall Effect and Field Effect Measurements on the Same Single Nanowire Nano Lett. 16 205-11

[31] Gutsche C, Regolin I, Blekker K, Lysov A, Prost W and Tegude F J 2009 Controllable p-type doping of GaAs nanowires during vapor-liquid-solid growth Journal of Applied Physics 105024305

[32] Stern E, Cheng G, Cimpoiasu E, Klie R, Guthrie S, Klemic J, Kretzschmar I, Steinlauf E, Turner-Evans D, Broomfield E, Hyland J, Koudelka R, Boone T, Young M, Sanders A, Munden R, Lee T, Routenberg D and Reed M A 2005 Electrical characterization of single GaN nanowires Nanotechnology 16 2941-2953

[33] Joyce H J, Boland J L, Davies C L, Baig S A and Johnston M B 2016 A review of the electrical properties of semiconductor nanowires: insights gained from terahertz conductivity spectroscopy Semicond. Sci. Technol. 31103003

[34] Ketterer B, Uccelli E and Morral A F i 2012 Mobility and carrier density in p-type GaAs nanowires measured by transmission Raman spectroscopy Nanoscale 4 1789-93

[35] Ketterer B, Mikheev E, Uccelli E and Fontcuberta i Morral A 2010 Compensation mechanism in silicon-doped gallium arsenide nanowires Appl. Phys. Lett. 97223103

[36] Goktas N I, Fiordaliso E M and LaPierre R R 2018 Doping assessment in GaAs nanowires Nanotechnology 29234001

[37] Chen H-L, Himwas C, Scaccabarozzi A, Rale P, Oehler F, Lemaître A, Lombez L, Guillemoles J-F, Tchernycheva M, Harmand J-C, Cattoni A and Collin S 2017 Determination of n-Type Doping Level in Single GaAs Nanowires by Cathodoluminescence Nano Lett. 17 6667-75

[38] Leamy H J 1982 Charge collection scanning electron microscopy Journal of Applied Physics 53 R51-80 
[39] Donolato C 1981 An analytical model of SEM and STEM charge collection images of dislocations in thin semiconductor layers: I. Minority carrier generation, diffusion, and collection physica status solidi (a) 65 649-58

[40] Tchoulfian P, Donatini F, Levy F, Dussaigne A, Ferret P and Pernot J 2014 Direct Imaging of $\mathrm{p}-\mathrm{n}$ Junction in Core-Shell GaN Wires Nano Lett. 14 3491-8

[41] Gutsche C, Niepelt R, Gnauck M, Lysov A, Prost W, Ronning C and Tegude F-J 2012 Direct Determination of Minority Carrier Diffusion Lengths at Axial GaAs Nanowire pn Junctions Nano Lett. 12 1453-8

[42] Pasemann L 1981 A contribution to the theory of the ebic contrast of lattice defects in semiconductors Ultramicroscopy 6 237-50

[43] Oehler F, Cattoni A, Scaccabarozzi A, Patriarche G, Glas F and Harmand J-C 2018 Measuring and Modeling the Growth Dynamics of Self-Catalyzed GaP Nanowire Arrays Nano Lett. 18 701-8

[44] Himwas C, Collin S, Chen H-L, Patriarche G, Oehler F, Travers L, Saket O, Julien F H, Harmand J-C and Tchernycheva M 2019 Correlated optical and structural analyses of individual GaAsP/GaP core-shell nanowires Nanotechnology 30304001

[45] Lépinau R de, Scaccabarozzi A, Patriarche G, Travers L, Collin S, Cattoni A and Oehler F 2019 Evidence and control of unintentional As-rich shells in GaAs1-x P x nanowires Nanotechnology 30294003

[46] Grassman T J, Brenner M R, Gonzalez M, Carlin A M, Unocic R R, Dehoff R R, Mills $\mathrm{M} J$ and Ringel S A 2010 Characterization of Metamorphic GaAsP/Si Materials and Devices for Photovoltaic Applications IEEE Trans. Electron Devices 57 3361-9

[47] Piazza V, Wirths S, Bologna N, Ahmed A A, Bayle F, Schmid H, Julien F and Tchernycheva M 2019 Nanoscale analysis of electrical junctions in InGaP nanowires grown by template-assisted selective epitaxy Appl. Phys. Lett. 114103101

[48] Anon nextnano - Software for semiconductor nanodevices

[49] Narchi P, Neplokh V, Piazza V, Bearda T, Bayle F, Foldyna M, Toccafondi C, Prod'homme P, Tchernycheva M and Roca i Cabarrocas P 2017 Surface potential investigation on interdigitated back contact solar cells by Scanning Electron Microscopy and Kelvin Probe Force Microscopy: Effect of electrical bias Solar Energy Materials and Solar Cells $161263-9$

[50] Barrigón E, Hultin O, Lindgren D, Yadegari F, Magnusson M H, Samuelson L, Johansson L I M and Björk M T 2018 GaAs Nanowire pn-Junctions Produced by LowCost and High-Throughput Aerotaxy Nano Lett. 18 1088-92

[51] Kurniawan O and Ong V K S 2009 Choice of Generation Volume Models for Electron Beam Induced Current Computation IEEE Transactions on Electron Devices 56 1094-9

[52] Ong V K S, Kurniawan O, Moldovan G and Humphreys C J 2006 A method of accurately determining the positions of the edges of depletion regions in semiconductor junctions Journal of Applied Physics 100114501 
[53] Norman C E 2001 Challenging the Spatial Resolution Limits of CL and EBIC SSP 7879 19-28

[54] Chang C-C, Chi C-Y, Yao M, Huang N, Chen C-C, Theiss J, Bushmaker A W, LaLumondiere S, Yeh T-W, Povinelli M L, Zhou C, Dapkus P D and Cronin S B 2012 Electrical and Optical Characterization of Surface Passivation in GaAs Nanowires Nano Lett. 12 4484-9

[55] Casadei A, Krogstrup P, Heiss M, Röhr J A, Colombo C, Ruelle T, Upadhyay S, Sørensen C B, Nygård J and Fontcuberta i Morral A 2013 Doping incorporation paths in catalyst-free Be-doped GaAs nanowires Appl. Phys. Lett. 102013117 